\title{
Donating convalescent plasma for COVID-19 patients
}

\author{
By Emily Black, Final Year Dental Student, University of Bristol Dental Hospital, Bristol, UK
}

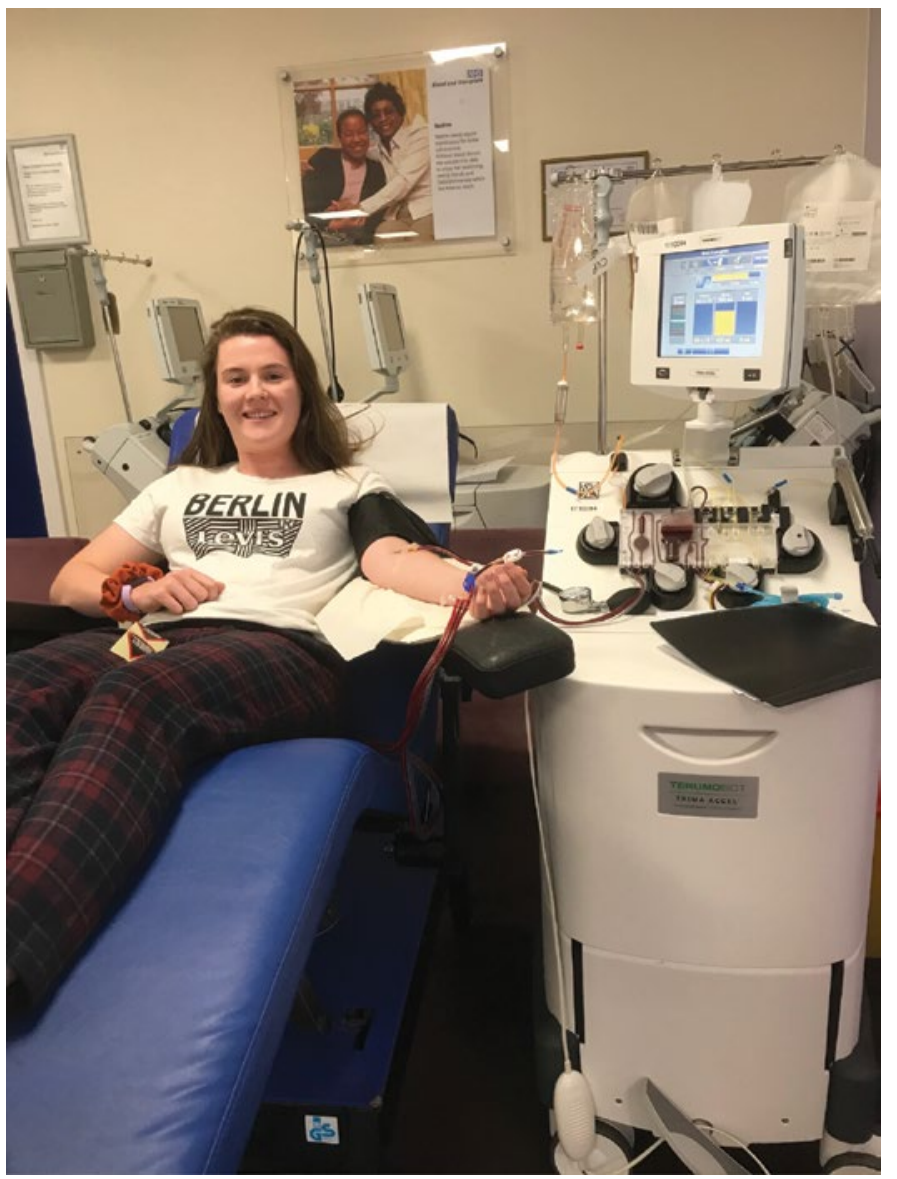

I write this piece as a call to any individual who has recovered from an infection with COVID-19. I tested positive back in March after falling ill following my return home from Bristol Dental School. After thankfully making a full recovery, and hearing that I wouldn't be returning to my clinical studies for six months, I decided to use the extra time on my hands to do what I could to help support the NHS during the pandemic in the United Kingdom.

After receiving the press release from the BDA urging dentists and dental team members to put themselves forward, I thought I would take this opportunity to share my experience. Within two weeks of applying through the NHS jobs website, I was in a classroom learning how to use telephone assessment protocols (NHS Pathways). This was in order to triage calls as an NHS 111 Health Advisor specifically for COVID-19 with the South-Central Ambulance Service NHS Foundation Trust (SCAS). After two weeks of one to one coaching, I went solo and was soon dispatching emergency ambulances to unwell coronavirus patients throughout England. During this time, I started to appreciate the great impact that the pandemic was having on the wider healthcare sector, as well as the general population.

In May, I was invited to volunteer to participate in a national NHS trial to help the sickest patients on intensive care units with COVID19. ${ }^{1}$ Those who had previous exposure to COVID-19 and had as a result high levels of neutralising antibodies could participate in the trial by donating their convalescent plasma. Having spoken to countless breathless COVID-19 patients on the NHS 111 Service, and as I was also aware of the high mortality rates for patients admitted into intensive care units, I immediately volunteered.

After being screened, UK Blood and Transplant confirmed that I had potentially therapeutic levels of antibodies. I was one of the first patients at my local plasma donation centre in Oxford to donate convalescent plasma as part of the UK trial in May 2020. I was actually surprised to find that donating my plasma was a much more pleasant experience than I had initially anticipated because donation does not reduce your circulating blood volume or red blood cell count. This also means you can potentially donate your plasma every two weeks, rather than every three months for whole blood. Every time I have donated, which takes approximately one hour, I have always been made to feel so welcome, comfortable, supported and valued by the donor carers and transfusion nursing staff. Since returning to university in September 2020 I have been able to continue donating my plasma in Bristol.

Clinical trials are currently taking place across the world looking at the therapeutic efficacy of both convalescent plasma and synthetically made COVID-19 antibodies. The recently published PLACID trial using human plasma in India has shown mixed results, although the study did not exclusively use plasma with high antibody levels, and participants were not blinded, so treatment status may have been influenced by subjective reporting of symptoms. ${ }^{2}$ Trials are still ongoing worldwide and if its therapeutic effectiveness is proven, the demand for human convalescent plasma will potentially be huge.

If you've had COVID-19, please consider donating your convalescent blood plasma. If you have not, then you could consider donating your much-needed blood or platelets. NHS Blood and Transplant are always looking for new donors. ${ }^{2}$

You could help save a life - please do what you can to help the NHS during these unprecedented times!

\section{References}

1. NHS Blood and Transplant. Convalescent Plasma - clinical trial. 2020. Available at: https://www.nhsbt.nhs.uk/how-you-can-help/convalescent-plasma-clinical-trial/ (accessed 17 November 2020).

2. Agarwal A, Mukherjee A, Kumar G, Chatterjee P, Bhatnagar T, Malhotra P Convalescent plasma in the management of moderate covid-19 in adults in India: open label phase II multicentre randomised controlled trial (PLACID Trial). BMJ 2020; 371: m3939. 